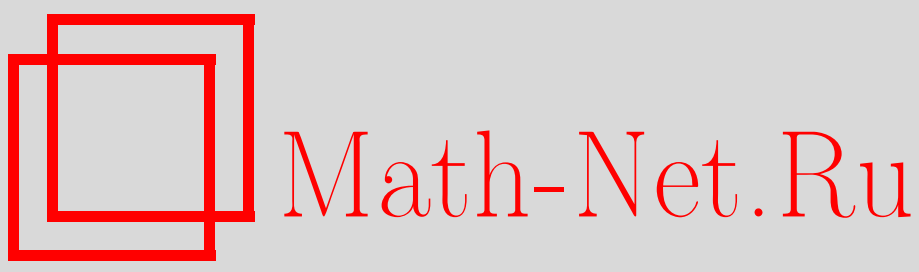

В. Г. Лысов, Сильная асимптотика аппроксимаций Эрмита-Паде для системы стилтьесовских функций с весом Лагерра, Матем. сб., 2005, том 196, номер 12, 99-122

DOI: https://doi.org/10.4213/sm1444

Использование Общероссийского математического портала Math-Net.Ru подразумевает, что вы прочитали и согласны с пользовательским соглашением

http: //www. mathnet.ru/rus/agreement

Параметры загрузки:

IP: 3.80 .253 .173

26 апреля 2023 г., 08:22:37 
УДК 517.53

\author{
В.Г. Лысов
}

\title{
Сильная асимптотика аппроксимаций Эрмита-Паде для системы стилтьесовских функций с весом Лагерра
}

Рассматриваются аппроксимации Эрмита-Паде с общим знаменателем для двух стилтьесовских функций с весами $x^{\alpha} e^{-\beta_{1} x}$ и $x^{\alpha} e^{-\beta_{2} x}$, где $\alpha>-1, \beta_{2}>$ $\beta_{1}>0$. Методом матричной задачи Римана-Гильберта получена сильная асимптотика этих аптроксимаций в случае $\beta_{2} / \beta_{1}<3+2 \sqrt{2}$. Показано, что предельное распределение нулей знаменателей аппроксимаций Эрмита-Паде есть равновесная мера для некоторой системы Никишина.

Библиограф̆ия: 11 названий.

\section{§1. Введение}

1.1. Постановка задачи. Пусть $\left(f_{1}, \ldots, f_{r}\right)$ - набор степенных рядов с центром в бесконечной точке:

$$
f_{j}(z)=\sum_{k=0}^{\infty} \frac{c_{j, k}}{z^{k+1}}, \quad j=1, \ldots, r .
$$

Аппроксимациями Эрмита-Паде (второго рода), соответствующими векторному индексу $\vec{n}=\left(n_{1}, \ldots, n_{r}\right) \in \mathbb{Z}_{+}^{r}$, называются рациональные функции с общим знаменателем

$$
\Pi_{\vec{n}, j}=\frac{p_{\vec{n}, j}}{q_{\vec{n}}}, \quad j=1, \ldots, r,
$$

такие, что

$$
\operatorname{deg} q_{\vec{n}} \leqslant n_{1}+\cdots+n_{r}
$$

И

$$
r_{\vec{n}, j}:=\left(q_{\vec{n}} f_{j}-p_{\vec{n}, j}\right)(z)=O\left(\frac{1}{z^{n_{j}+1}}\right), \quad j=1, \ldots, r .
$$

Исследование сходимости и асимптотического поведения аппроксимаций (1.2) является сложной задачей с богатым содержанием. В работе А.А. Гончара и Е. А. Рахманова [1] была изучена сходимость аппроксимаций для системы марковских функций

$$
f_{j}(z)=\frac{1}{2 \pi i} \int_{\Delta_{j}} \frac{d \sigma_{j}(x)}{x-z}, \quad j=1, \ldots, r,
$$

Работа вьполнена при поддержке Российского фонда фундаментальных исследований (грант № 05-01-00522), Программы поддержки ведущих научных школ Р $\Phi$ (грант № НШ-1551.2003.1), Отделения математических наук РАН (программа № 1) и фонда INTAS (грант № 03-516637). 
где $\Delta_{j}$ - попарно не пересекающиеся отрезки на вешественной оси. Одним из главных выводов работы [1] явилось то, что сходимость, расходимость, предельное распределение полюсов существенно зависят от “геометрии” особенностей функций (1.5) (т.е. расположения отрезков $\Delta_{j}$ ).

В настоящей работе мы рассматриваем асимптотику аппроксимаций ЭрмитаПаде для системы функций стилтьесовского типа

$$
f_{j}(z)=\frac{1}{2 \pi i} \int_{0}^{\infty} \frac{w_{j}(x) d x}{x-z}, \quad j=1, \ldots, r,
$$

где $\left(w_{1}, \ldots, w_{r}\right)$ - система весов Лагерра:

$$
w_{j}(x)=x^{\alpha} e^{-\beta_{j} x}, \quad j=1, \ldots, r, \quad \alpha>-1, \quad \beta_{r}>\cdots>\beta_{1}>0 .
$$

При $r=1$ знаменатели $l_{\vec{n}}:=q_{\vec{n}}$ являются классическими полиномами Лагерра. При $r>1$ эти полиномы являются полиномами совместной ортогональности:

$$
\int_{0}^{\infty} l_{\vec{n}}(x) x^{k} w_{j}(x) d x=0, \quad k=0, \ldots, n_{j}-1, \quad j=1, \ldots, r .
$$

Известно (см. [2]), что полином $l_{\vec{n}}$ определен однозначно с точностью до нормировочной постоянной. Все нули $l_{\vec{n}}$ простые и лежат в интервале $(0, \infty)$. Аналогично классическим полиномы совместной ортогональности Лагерра могут быть представлены формулой Родрига (см. [2; гл. $4, \S 6])$ :

$$
l_{\vec{n}}(x)=x^{-\alpha} \prod_{j=1}^{r}\left(1-\frac{1}{\beta_{j}} \frac{d}{d x}\right)^{n_{j}} x^{|\vec{n}|+\alpha},
$$

где произведение дифференциальных операторов можно брать в любом порядке, и старший коэффициент $l_{\vec{n}}$ равен единице.

Алгебраические свойства полиномов совместной ортогональности для классических весов, удовлетворяющих уравнению Пирсона, недавно были изучены в работе [3]. В частности, там были получены рекуррентные соотношения и линейное дифференциальное уравнение для полиномов совместной ортогональности Лагерpa $l_{\vec{n}}(x)$.

Вопросы сходимости аппроксимаций Эрмита-Паде для системы $(1.6),(1.7)$ были рассмотрены В.Н. Сорокиным в [4]. Свое внимание мы сконцентрируем на асимптотическом поведении $l_{\vec{n}}, r_{\vec{n}, j}$, в частности на описании предельного поведения полюсов аппроксимаций. Как уже отмечалось выше, нули $l_{\vec{n}}$ плотны в $[0, \infty]$. Для более точного описания их предельного распределения полезно изменить масштаб задачи, так чтобы они заметали компакте. В настоящей работе изучается асимптотика масштабированных полиномов с единичным старшим коэффициентом:

$$
L_{n}(x)=n^{-2 n} l_{\vec{n}}(n x),
$$

в случае двух весов $(r=2)$ и диагональных индексов $(n, n)$. Полиномы $L_{n}(x)$ удовлетворяют соотношениям ортогональности с переменным весом

$$
\int_{0}^{\infty} L_{n}(x) x^{k+\alpha} e^{-n \beta_{1} x} d x=0, \quad \int_{0}^{\infty} L_{n}(x) x^{k+\alpha} e^{-n \beta_{2} x} d x=0 .
$$

Рассматривается случай малого отношения параметров $\beta_{2} / \beta_{1}<3+2 \sqrt{2}$.

Автор выражает благодарность В.Н. Сорокину за постановку задачи и А. И. Аптекареву за полезные обсуждения в процессе работы. 
1.2. Риманова поверхность. В задачах об асимптотике аппроксимаций Эрмита-Паде ключевую роль играют алгебраические функции, определяюшие главньй член асимптотики (см. [5]). В этом пункте мы опишем соответствующую алгебраическую функцию и ее риманову поверхность. Предполагаем, что после сжатия плоскости нули полиномов $L_{n}(x)$ заметают некоторый компакт и можно ожидать асимптотику вне этого компакта в виде

$$
L_{n}(z)=F(z) \Phi^{n}(z)\left(1+O\left(\frac{1}{n}\right)\right), \quad n \rightarrow \infty,
$$

где $\Phi(z)$ является ветвью некоторой трехзначной аналитической функции. Явный вид этой функции можно найти следующим образом. Используя результаты работы [3], после некоторых преобразований получаем дифференциальное уравнение, которому удовлетворяет полином $L_{n}(z)$ :

$$
\begin{aligned}
& z^{2} L_{n}^{\prime \prime \prime}(z)-z\left[n z\left(\beta_{1}+\beta_{2}\right)-2(\alpha+1)\right] L_{n}^{\prime \prime}(z) \\
& \quad+\left[\beta_{1} \beta_{2} n^{2} z^{2}+\left(\beta_{1}+\beta_{2}\right)(\alpha+1-n) n z+\alpha(\alpha+1)\right] L_{n}^{\prime}(z) \\
& \quad-n^{2}\left[2 \beta_{1} \beta_{2} n z+\alpha\left(\beta_{1}+\beta_{2}\right)\right] L_{n}(z)=0 .
\end{aligned}
$$

Подставляя асимптотическую формулу в это уравнение и приравнивая коэффициенты при старшем члене по $n$, получаем алгебраическое уравнение для логарифмической производной $\psi(z)=\Phi^{\prime} / \Phi(z)$ функции $\Phi$ :

$$
z \psi^{3}(z)-\left(\beta_{1}+\beta_{2}\right) z \psi^{2}(z)+\left(\beta_{1} \beta_{2} z+\beta_{1}+\beta_{2}\right) \psi(z)-2 \beta_{1} \beta_{2}=0,
$$

или равносильно

$$
z=\frac{2}{\psi(z)}-\frac{1}{\psi(z)-\beta_{1}}-\frac{1}{\psi(z)-\beta_{2}} .
$$

Алгебраическая функция $\psi(z)$ допускает выделение трех голоморфных ветвей при $z \rightarrow \infty$ :

$$
\begin{aligned}
& \psi_{0}(z)=\frac{2}{z}+O\left(\frac{1}{z^{2}}\right) \\
& \psi_{1}(z)=\beta_{1}-\frac{1}{z}+O\left(\frac{1}{z^{2}}\right) \\
& \psi_{2}(z)=\beta_{2}-\frac{1}{z}+O\left(\frac{1}{z^{2}}\right) .
\end{aligned}
$$

Найдем теперь выражение для $\Phi$ :

$$
\Phi(z)=c_{0} e^{\int \psi d z}=c_{0} e^{z \psi-\int z d \psi}=c_{0} e^{z \psi}\left(\psi-\beta_{1}\right)\left(\psi-\beta_{2}\right) \psi^{-2} .
$$

Выберем постоянную $c_{0}=\left(\beta_{1} \beta_{2} e^{2} / 4\right)^{-1}$. Тогда соответствующие ветви функции $\Phi$ имеют следуюшее поведение в окрестности бесконечности:

$$
\begin{aligned}
& \Phi_{0}(z)=z^{2}\left(1+O\left(\frac{1}{z}\right)\right), \\
& \Phi_{1}(z)=c_{1} e^{\beta_{1} z} \frac{1}{z}\left(1+O\left(\frac{1}{z}\right)\right), \\
& \Phi_{2}(z)=c_{2} e^{\beta_{2} z} \frac{1}{z}\left(1+O\left(\frac{1}{z}\right)\right),
\end{aligned}
$$


где

$$
c_{1}=\left(\frac{\beta_{1}^{3} \beta_{2} e^{3}}{4\left(\beta_{2}-\beta_{1}\right)}\right)^{-1}, \quad c_{2}=\left(\frac{\beta_{2}^{3} \beta_{1} e^{3}}{4\left(\beta_{1}-\beta_{2}\right)}\right)^{-1}
$$

Нас интересует множество, где равны по модулю различные ветви функции $\Phi$ : $M=\left\{z \in \mathbb{C}:\left|\Phi_{j}(z)\right|=\left|\Phi_{k}(z)\right|, j \neq k\right\}$.

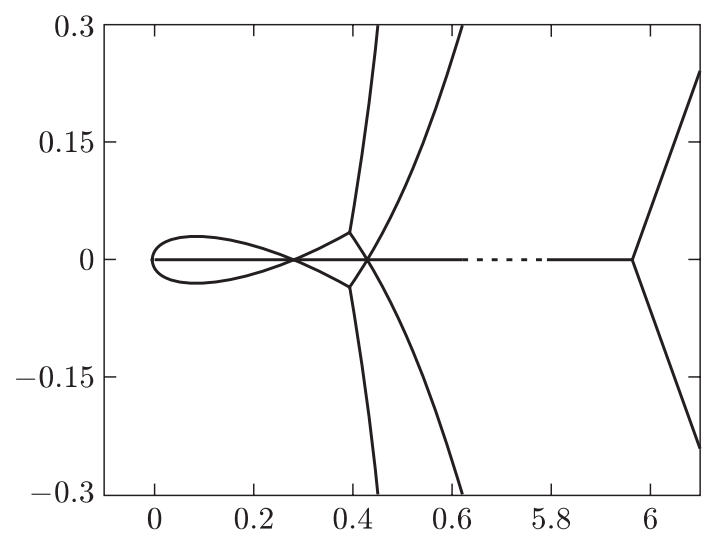

Рис. 1. Множество $M$ при $\varkappa_{N}<\beta_{2} / \beta_{1}<\varkappa_{A}$

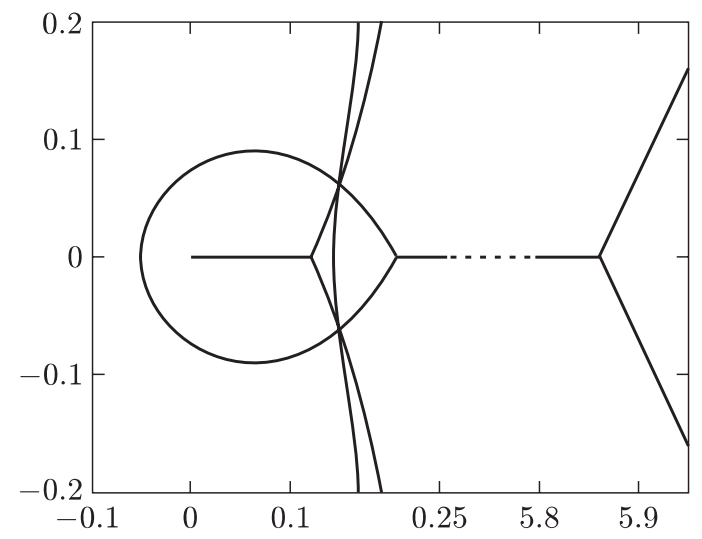

Рис. 2. Множество $M$ при $\beta_{2} / \beta_{1}>\varkappa_{A}$

Структура этого множества зависит от отношения $\beta_{2} / \beta_{1}$ (см. рис. $\left.1-4\right)$. Введем обозначения

$$
\varkappa_{N}=3+2 \sqrt{2}, \quad \varkappa_{A}=\frac{7+3 \sqrt{3}}{2}+\sqrt{\frac{36+21 \sqrt{3}}{2}}=12.1136 \ldots
$$

При $\beta_{2} / \beta_{1}<\varkappa_{A}$ функция $\Phi(z)$ имеет четыре простые точки ветвления, две из которых вещественные 0 и $d(d>0)$, другие две комплексно сопряженные $c$ и $\bar{c}$ 


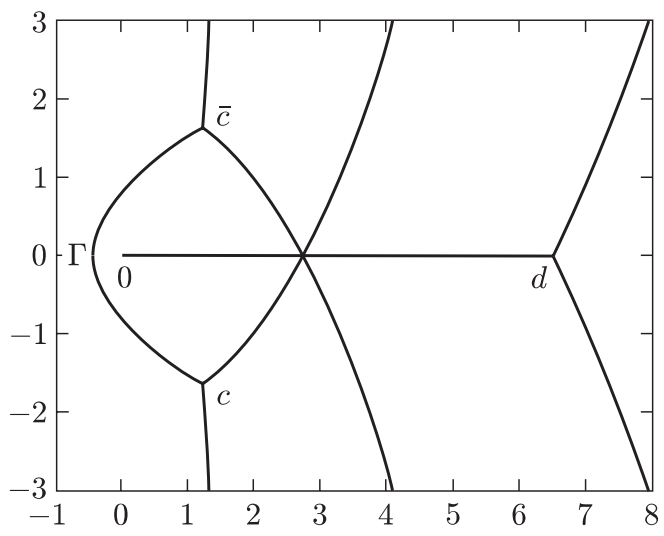

Рис. 3. Множество $M$ при $\varkappa_{N N}<\beta_{2} / \beta_{1}<\varkappa_{N}$

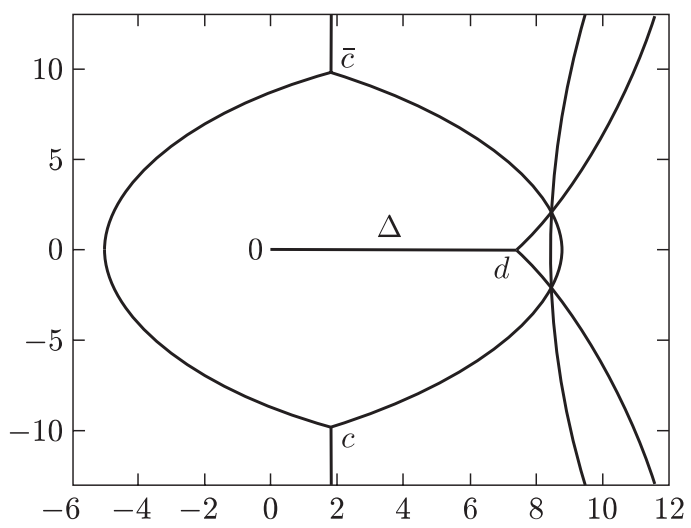

Рис. 4. Множество $M$ при $\beta_{2} / \beta_{1}<\varkappa_{N N}$

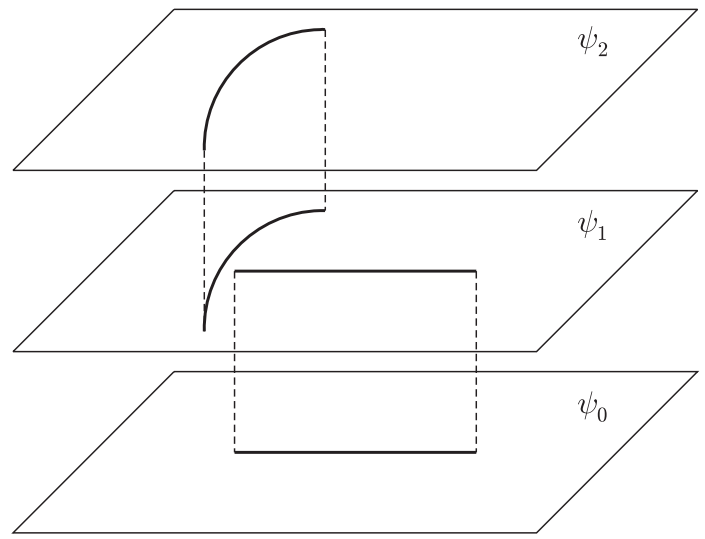

Рис. 5. Риманова поверхность функции $\psi$ 
$(\operatorname{Im} c<0)$. При $\beta_{2} / \beta_{1}<\varkappa_{N}$ множеству $\left\{z \in \mathbb{C}:\left|\Phi_{j}(z)\right|=\left|\Phi_{k}(z)\right|, j \neq k\right\}$ принадлежит простая аналитическая кривая $\Gamma$, соединяющая точки $c$ и $\bar{c}$ и пересекаюшая действительную ось в отрицательной точке, а также отрезок $\Delta=[0, d]$. Делая разрезы вдоль этих кривых, выделяем голоморфные ветви функции $\psi($ и $\Phi)$ :

$$
\begin{aligned}
& \psi_{0}(z) \in H(\mathbb{C} \backslash \Delta), \\
& \psi_{1}(z) \in H(\mathbb{C} \backslash \Delta \cup \Gamma), \\
& \psi_{2}(z) \in H(\mathbb{C} \backslash \Gamma) .
\end{aligned}
$$

Риманова поверхность функции $\psi$ изображена на рис. 5 .

1.3. Формулировка результатов. Как будет показано ниже, в случае малого отношения параметров нули полиномов $L_{n}(x)$ заметают отрезок $\Delta$. Мы получим асимптотические формулы для $L_{n}(x)$ вне $\Delta$, внутри $\Delta$ и в окрестности концов $\Delta$.

Теорема 1.1. Для полиномов совместной ортогональности Лагерра $L_{n}(z)$ в случае $\beta_{2} / \beta_{1}<3+2 \sqrt{2}$ справедливы следующие асимптотические формулы nрu $n \rightarrow \infty$ :

1) равномерно на компактных подмножествах $\mathbb{C} \backslash \Delta$

$$
L_{n}(z)=F\left(z, \psi_{0}(z)\right) \Phi_{0}^{n}(z)\left(1+O\left(\frac{1}{n}\right)\right)
$$

əде

$$
F(z, \psi)=\frac{2^{\alpha} \psi^{-\alpha} z^{-\alpha}\left(\psi-\beta_{1}\right)\left(\psi-\beta_{2}\right)}{\sqrt{D(\psi)}},
$$

$D(\psi)=-4\left(\beta_{1}+\beta_{2}\right) \psi^{3}+2\left(\beta_{1}^{2}+8 \beta_{1} \beta_{2}+\beta_{2}^{2}\right) \psi^{2}-8\left(\beta_{1}+\beta_{2}\right) \beta_{1} \beta_{2} \psi+4 \beta_{1}^{2} \beta_{2}^{2} ;$

2) равномерно на компактных подмножсествах $(0, d)$

$$
L_{n}(x)=\left(F\left(x, \psi_{0+}(x)\right) \Phi_{0+}^{n}(x)+F\left(x, \psi_{0-}(x)\right) \Phi_{0-}^{n}(x)\right)\left(1+O\left(\frac{1}{n}\right)\right) ;
$$

3) равномерно в достаточно малой окрестности точки 0

$$
\begin{aligned}
L_{n}(z)=( & \left.\Phi_{1} \Phi_{0}\right)^{n / 2} \sqrt{-\pi n \varphi}\left(I_{\alpha}\left(-\frac{n \varphi}{2}\right)\left(N_{00}(z)+i N_{01}(z)(-z)^{-\alpha}\right)\right. \\
& \left.+I^{\prime}{ }_{\alpha}\left(-\frac{n \varphi}{2}\right)\left(-N_{00}(z)+i N_{01}(z)(-z)^{-\alpha}\right)\right)\left(1+O\left(\frac{1}{n}\right)\right) ;
\end{aligned}
$$

4) равномерно в достаточно малой окрестности точки d

$$
\begin{aligned}
L_{n}(z)=( & \left.\Phi_{1} \Phi_{0}\right)^{n / 2} \sqrt{\pi}\left(\left(\frac{3}{2} n \varphi\right)^{1 / 6} \operatorname{Ai}\left(\left(\frac{3}{2} n \varphi\right)^{2 / 3}\right)\left(N_{00}(z)+i N_{01}(z) z^{-\alpha}\right)\right. \\
& \left.+\left(\frac{3}{2} n \varphi\right)^{-1 / 6} \operatorname{Ai}^{\prime}\left(\left(\frac{3}{2} n \varphi\right)^{2 / 3}\right)\left(-N_{00}(z)+i N_{01}(z) z^{-\alpha}\right)\right) \\
& \times\left(1+O\left(\frac{1}{n}\right)\right)
\end{aligned}
$$




$$
\begin{gathered}
\text { əде } \varphi=\ln \left(\Phi_{1} / \Phi_{0}\right), I_{\alpha}(\zeta)-\text { функиия Бесселя, } \operatorname{Ai}(\zeta)-\text { функция Эйри, } \\
N_{00}(z)=\frac{2^{\alpha}\left(\psi_{0}-\beta_{1}\right)\left(\psi_{0}-\beta_{2}\right) \psi_{0}^{-\alpha} z^{-\alpha}}{\sqrt{D\left(\psi_{0}\right)}} \\
N_{01}(z)=\frac{2^{\alpha}\left(\psi_{1}-\beta_{1}\right)\left(\psi_{1}-\beta_{2}\right) \psi_{1}^{-\alpha}}{\sqrt{D\left(\psi_{1}\right)}}
\end{gathered}
$$

Теорема будет доказана методом, основанным на рассмотрении некоторой матричной краевой задачи Римана-Гильберта. Этот метод изучения асимптотики ортогональных многочленов был предложен П. Дейфтом и Х. Чжу в работе [6]. Для многочленов совместной ортогональности соответствуюшая задача РиманаГильберта была сформулирована в работе [7]. В этой работе мы используем задачу Римана-Гильберта, естественную для полиномов совместной ортогональности для системы Никишина.

\section{§2. Критические траектории и равновесные меры}

В этом параграфе мы докажем необходимые свойства кривых, принадлежащих множеству $M$, и рассмотрим равновесные меры на них.

2.1. Критические траектории. Из определения $\psi$ легко видеть, что множество $M$ есть объединение критических траекторий квадратичных дифференциалов $\left(\psi_{j}-\psi_{k}\right)^{2} d z^{2}$, т.е. траекторий, выходящих из нулей или полюсов $\left(\psi_{j}-\psi_{k}\right)^{2}$ (точек ветвления $\psi$ ), таких, что $\left(\psi_{j}-\psi_{k}\right)^{2} d z^{2}<0$. Трехзначная алгебраическая функция $\psi(z)$ имеет четыре простые точки ветвления: две вешественные 0 и $d$ $(d>0)$ и две комплексно сопряженные $c$ и $\bar{c}(\operatorname{Im} c<0)$. Эти точки являются значениями рациональной функции $z(\psi)$ в критических точках $\left(z^{\prime}(\psi)=0\right) \infty, \psi_{d}, \psi_{c}, \psi_{\bar{c}}$, или равносильно нулями дискриминанта

$$
\begin{aligned}
& \beta_{1}^{2} \beta_{2}^{2}\left(\beta_{1}-\beta_{2}\right)^{2} z^{4}-6 \beta_{1} \beta_{2}\left(\beta_{1}-\beta_{2}\right)^{2}\left(\beta_{1}+\beta_{2}\right) z^{3} \\
& \quad+\left(\beta_{1}^{4}+28 \beta_{1}^{3} \beta_{2}-54 \beta_{1}^{2} \beta_{2}^{2}+28 \beta_{1} \beta_{2}^{3}+\beta_{2}^{4}\right) z^{2}-4\left(\beta_{1}+\beta_{2}\right)^{3} z .
\end{aligned}
$$

Чтобы выделить голоморфные ветви функции $\psi(z)$, в плоскости $\mathbb{C}$ сделаем два разреза $\Delta$ и $\Gamma$, где $\Delta$ есть отрезок $[0, d]$, а $\Gamma$ - жорданова кривая, соединяюшая точки $c$ и $\bar{c}$ и пересекаюшая вешественную ось один раз (в отрицательной точке). Ниже мы однозначно определим эту кривую, а пока считаем ее произвольной. Пронумеруем три ветви функции $\psi(z)$ согласно их поведению в окрестности точки $z=\infty$ :

$$
\begin{aligned}
& \psi_{0}(z)=\frac{2}{z}+O\left(\frac{1}{z^{2}}\right) \\
& \psi_{1}(z)=\beta_{1}-\frac{1}{z}+O\left(\frac{1}{z^{2}}\right) \\
& \psi_{2}(z)=\beta_{2}-\frac{1}{z}+O\left(\frac{1}{z^{2}}\right) .
\end{aligned}
$$


Тогда соответствующие ветви функции $\Phi(z)$ будут иметь следующую асимптотику в окрестности бесконечности:

$$
\begin{aligned}
& \Phi_{0}(z)=z^{2}\left(1+O\left(\frac{1}{z}\right)\right), \\
& \Phi_{1}(z)=c_{1} e^{\beta_{1} z} \frac{1}{z}\left(1+O\left(\frac{1}{z}\right)\right), \\
& \Phi_{2}(z)=c_{2} e^{\beta_{2} z} \frac{1}{z}\left(1+O\left(\frac{1}{z}\right)\right),
\end{aligned}
$$

где

$$
c_{1}=\left(\frac{\beta_{1}^{3} \beta_{2} e^{3}}{4\left(\beta_{2}-\beta_{1}\right)}\right)^{-1}, \quad c_{2}=\left(\frac{\beta_{2}^{3} \beta_{1} e^{3}}{4\left(\beta_{1}-\beta_{2}\right)}\right)^{-1} .
$$

Как легко видеть из графика рациональной функции $z(\psi)$ при вещественных $\psi$ (см. рис. 6), эти ветви допускают голоморфные продолжения в следующие области:

$$
\begin{aligned}
& \psi_{0}, \Phi_{0} \in H(\mathbb{C} \backslash \Delta), \\
& \psi_{1}, \Phi_{1} \in H(\mathbb{C} \backslash(\Delta \cup \Gamma)), \\
& \psi_{2}, \Phi_{2} \in H(\mathbb{C} \backslash \Gamma) .
\end{aligned}
$$

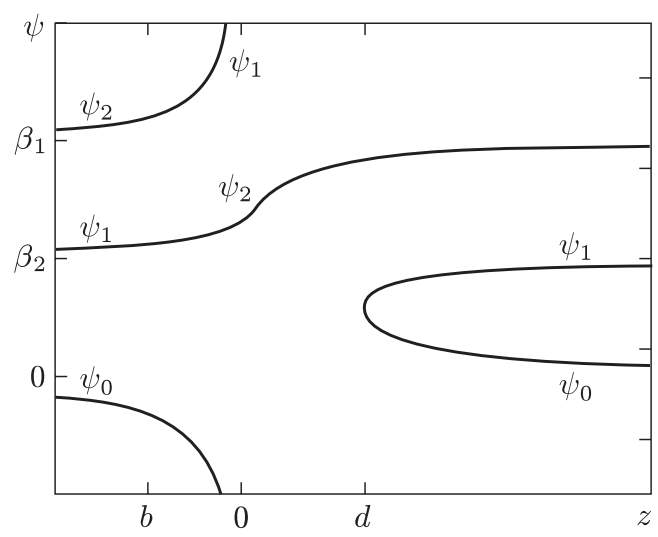

Рис. 6. Граффик ветвей функции $\psi(z), z \in \mathbb{R}$

В точке ветвления $z=0$ функция $\psi(z)$ имеет полюс и разложение ветвей имеет следуюший вид:

$$
\begin{aligned}
& \psi_{0}(z)=-\frac{i \sqrt{\beta_{1}+\beta_{2}}}{\sqrt{z}}+\frac{1}{2} \frac{\beta_{1}^{2}+\beta_{2}^{2}}{\beta_{1}+\beta_{2}}+O(\sqrt{z}), \\
& \psi_{1}(z)=\frac{i \sqrt{\beta_{1}+\beta_{2}}}{\sqrt{z}}+\frac{1}{2} \frac{\beta_{1}^{2}+\beta_{2}^{2}}{\beta_{1}+\beta_{2}}+O(\sqrt{z}), \\
& \psi_{2}(z)=\frac{2 \beta_{1} \beta_{2}}{\beta_{1}+\beta_{2}}+O(z) .
\end{aligned}
$$

Нам понадобятся две леммы. 
Лемма 2.1. Множество $\left\{x \in \mathbb{R}:\left|\Phi_{1}(x)\right|=\left|\Phi_{2}(x)\right|\right\}$ состоит из двух точек $b_{-} u b_{+} . \Pi р и$ этом $b_{-}<0, a b_{+}>0$.

ДокаЗАтельство. Рассмотрим функцию $\varphi_{21}(x):=\ln \left|\Phi_{2} / \Phi_{1}\right|(x)$. Из асимптотики $\Phi_{1}, \Phi_{2}$ в окрестности бесконечности $(2.6),(2.7)$ следует, что

$$
\varphi_{21}(+\infty)=+\infty, \quad \varphi_{21}(-\infty)=-\infty .
$$

Можно выбрать разрез Г так, чтобы он пересекал вешественную ось в точке $b<0$ такой, что

$$
\varphi_{21}(x)<0, \quad x \in(-\infty, b) .
$$

На $(b,+\infty)$ функция $\varphi_{21}$ непрерывна. На интервале $(b, 0)$ функция $\varphi_{21}(x)$ монотонно убывает: $\varphi_{21}^{\prime}=\psi_{2}-\psi_{1}<0$, а в концах этого интервала принимает значения разных знаков:

$$
\begin{gathered}
\varphi_{21}(b+)=-\varphi_{21}(b-)>0, \\
\varphi_{21}(0)=\ln \frac{(\beta-1)^{2}(\beta+1)^{2}}{4 \beta}<0, \quad \beta<3+2 \sqrt{2} .
\end{gathered}
$$

Поэтому существует единственная точка $b_{-}<0$ такая, что $\varphi_{21}\left(b_{-}\right)=0$. Далее, так как $\varphi_{21}(+\infty)=+\infty$, то сушествует точка $b_{+}>0: \varphi_{21}\left(b_{+}\right)=0$. Так как на отрезке $(0, d) \quad \varphi_{21}$ выпукла: $\varphi_{21}^{\prime \prime}=\left(\psi_{2}-\operatorname{Re} \psi_{1}\right)^{\prime}=3 \psi_{2}^{\prime} / 2>0$, а на $(d, \infty)$ возрастает, то точка $b_{+}$единственна.

ЗАмЕчАниЕ 2.2. Нетрудно показать, что сушествует единственная постоянная $\varkappa_{N N}=1.26 \ldots$ такал, что если $\beta_{2} / \beta_{1}>\varkappa_{N N}$, то $b_{+}<d$, а если $\beta_{2} / \beta_{1}<\varkappa_{N N}$, то $b_{+}>d$. Критическое значение $\varkappa_{N N}$ определяется из условия $\varphi_{21}(d)=0$ однозначно, поскольку производная $\varphi_{21}(d)$ по отношению $\beta_{2} / \beta_{1}$ положительна.

Аналогично доказывается следуюшая лемма.

Лемма 2.3. Множество $\left\{x \in \mathbb{R}:\left|\Phi_{0}(x)\right|=\left|\Phi_{1}(x)\right|\right\}$ совпадает с отрезком $\Delta$. При $x>d\left|\Phi_{1}(x)\right|>\left|\Phi_{0}(x)\right|$.

Изучим критические траектории квадратичного дифференциала $\left(\psi_{2}-\psi_{1}\right)^{2} d z^{2}$ в верхней полуплоскости. Этот дифференциал имеет ровно одну особую точку $c$, которая является простьм нулем. Поэтому из нее выходят три траектории. Эти траектории не имеют других точек пересечения в замькании верхней полуплоскости (иначе противоречие с принципом максимума) и могут заканчиваться либо в бесконечности, либо на вещественной прямой. Согласно лемме 2.3 на вещественной оси есть только две точки $b_{-}$и $b_{+}$, в которых могут заканчиваться траектории. Значит, из трех траекторий, выходящих из $c$, по одной идет в каждую из точек $\infty, b_{-}, b_{+}$. В силу комплексной сопряженности ветвей $\psi$ поведение траекторий в нижней полуплоскости симметрично относительно вещественной оси. Таким образом, множество $\left\{z \in \mathbb{C}:\left|\Phi_{1}(z)\right|=\left|\Phi_{2}(z)\right|\right\}$ описано. Пользуясь разложением $\Phi$ в окрестности бесконечности, нетрудно восстановить знак выражения $\left|\Phi_{1}(z)\right|-\left|\Phi_{2}(z)\right|$ в каждой из трех областей, на которые критические траектории разбивают комплексную плоскость: $\left|\Phi_{1}(z)\right|<\left|\Phi_{2}(z)\right|$ в области, расположенной справа. 
2.2. Равновесные меры. Определим две меры на отрезке $\Delta$ и кривой $\Gamma$ соответственно:

$$
\begin{array}{ll}
\lambda(\zeta)=\frac{1}{2 \pi} \operatorname{Arg}\left(\Phi_{1}-\Phi_{0}\right)_{+}(\zeta), & \zeta \in \Delta, \\
\mu(\zeta)=\frac{1}{2 \pi} \operatorname{Arg}\left(\Phi_{2}-\Phi_{1}\right)_{+}(\zeta), & \zeta \in \Gamma .
\end{array}
$$

ПРЕДЛОЖЕНИЕ 2.4. Функции $\lambda$ и положительных мер на $\Delta$ и Г. Масса меры $\lambda$ равна 2 , масса меры $\mu$ равна 1.

ДокАЗАТЕЛЬСТво. Проведем доказательство для меры $\mu$. Вначале покажем, что функция $\mu$ монотонна вдоль ориентированной кривой $Г$. Действительно, производная $\mu$ вдоль кривой Г отлична от нуля:

$$
\frac{d \mu(\zeta)}{d \zeta}=\frac{1}{2 \pi i} \frac{d}{d \zeta}\left(\ln \Phi_{2}-\ln \Phi_{1}\right)_{+}(\zeta)=\frac{1}{2 \pi i}\left(\psi_{2}-\psi_{1}\right)_{+}(\zeta) \neq 0
$$

Для нахождения полной вариации меры $\mu$ воспользуемся последней формулой:

$$
\int_{\Gamma} d \mu(\zeta)=\frac{1}{2 \pi i} \int_{\Gamma}\left(\psi_{2}-\psi_{1}\right)_{+}(\zeta) d \zeta=\frac{1}{2 \pi i} \int_{\Gamma}\left(\psi_{2}^{+}-\psi_{2}^{-}\right)(\zeta) d \zeta
$$

так как в силу условий склейки $\psi_{1}^{+}=\psi_{2}^{-}$на кривой $Г$. Далее, рассмотрим контур $\delta$, обходящий кривую $Г$ по часовой стрелке. Тогда

$$
\frac{1}{2 \pi i} \int_{\Gamma}\left(\psi_{2}^{+}-\psi_{2}^{-}\right)(\zeta) d \zeta=\frac{1}{2 \pi i} \int_{\delta} \psi_{2}(z) d z=\operatorname{Res}_{\infty} \psi_{2}(z)=1
$$

по теореме Коши о вычетах.

Аналогично доказывается утверждение для $\lambda$.

Рассмотрим теперь комплексные логарифмические потенциалы мер $\lambda$ и $\mu$ :

$$
\begin{aligned}
& \mathscr{V}_{\lambda}(z)=\int_{\Delta} \ln \frac{1}{\zeta-z} d \lambda(\zeta), \\
& \mathscr{V}_{\mu}(z)=\int_{\Gamma} \ln \frac{1}{\zeta-z} d \mu(\zeta),
\end{aligned}
$$

определенные в $\mathbb{C} \backslash \Delta$ и $\mathbb{C} \backslash \Gamma$ по модулю $4 \pi i$ и $2 \pi i$ соответственно. Данные потенциалы выражаются через ветви функции $\Phi$.

ПРЕДЛОЖЕНИЕ 2.5. Комплексные потенциаль $\mathscr{V}_{\lambda}$ и $\mathscr{V}_{\mu}$ имеют явные представления в терминах $\Phi$ :

$$
\mathscr{V}_{\lambda}(z)=-\ln \Phi_{0}, \quad z \in \mathbb{C} \backslash \Delta,
$$

$u$

$$
\mathscr{V}_{\mu}^{\prime}(z)=\ln \Phi_{2}(z)-\beta_{2} z-\ln c_{2}, \quad z \in \mathbb{C} \backslash \Gamma .
$$


ДокАЗАТЕЛЬСТво. Снова проведем доказательство только для потенциала меры $\mu$. Производная $\mathscr{V}_{\mu}$ при $z \in \mathbb{C} \backslash \Gamma$ равна

$$
\mathscr{V}_{\mu}^{\prime}(z)=\frac{1}{2 \pi i} \int_{\Gamma} \frac{\left(\psi_{2}-\psi_{1}\right)_{+}(\zeta)}{\zeta-z} d \zeta=\frac{1}{2 \pi i} \int_{\delta} \frac{\psi_{2}(\zeta)}{\zeta-z} d \zeta
$$

где $\delta$ - контур, обходящий $Г$ по часовой стрелке и такой, что точка $z$ находится слева от $\delta$. Далее,

$$
\mathscr{V}_{\mu}^{\prime}(z)=\frac{1}{2 \pi i} \int_{\delta} \frac{\psi_{2}(\zeta)}{\zeta-z} d \zeta=\operatorname{Res}_{z} \frac{\psi_{2}(\zeta)}{\zeta-z}+\operatorname{Res}_{\infty} \frac{\psi_{2}(\zeta)}{\zeta-z}=\psi_{2}(z)-\beta_{2} .
$$

Интегрируя последнее выражение и учитывая поведение $\Phi$ в бесконечности, получаем требуемое.

ПРЕДЛОЖЕНИЕ 2.6. При $z \in \mathbb{C} \backslash(\Delta \cup \Gamma)$ имеем

$$
\begin{aligned}
& 2 \mathscr{V}_{\lambda}(z)-\mathscr{V}_{\mu}(z)=\ln \frac{\Phi_{1}}{\Phi_{0}}(z)-\beta_{1} z-\ln c_{1}, \\
& 2 \mathscr{V}_{\mu}(z)-\mathscr{V}_{\lambda}(z)=\ln \frac{\Phi_{2}}{\Phi_{1}}(z)-\left(\beta_{2}-\beta_{1}\right) z-\ln \frac{c_{2}}{c_{1}} .
\end{aligned}
$$

ЗАмЕЧАНИЕ 2.7. Из соотношений $(2.27),(2.28)$ следует, что $(\lambda, \mu)$ - векторная равновесная мера для системы Никишина с внешним полем $\left(\beta_{1} \operatorname{Re} z,\left(\beta_{2}-\beta_{1}\right) \operatorname{Re} z\right)$, т.е. их логарифмические потенциалы $(V:=\operatorname{Re} \mathscr{V})$ удовлетворяют следующим условиям равновесия:

$$
\begin{aligned}
2 V^{\lambda}(z)-V^{\mu}(z)+\beta_{1} z & =-\ln \left|c_{1}\right|, & & z \in \Delta, \\
2 V^{\mu}(z)-V^{\lambda}(z)+\left(\beta_{2}-\beta_{1}\right) \operatorname{Re} z & =\ln \left|c_{1}\right|-\ln \left|c_{2}\right|, & & z \in \Gamma .
\end{aligned}
$$

Подробнее о векторных задачах равновесия см. [2] и [8].

ЗАмЕчАниЕ 2.8. Легко видеть, что условия, наложенные нами на кривые $\Delta$ и $\Gamma$ :

$$
\begin{aligned}
& \left|\Phi_{0}(z)\right|=\left|\Phi_{1}(z)\right|, \quad z \in \Delta, \\
& \left|\Phi_{1}(z)\right|=\left|\Phi_{2}(z)\right|, \quad z \in \Gamma,
\end{aligned}
$$

эквивалентны свойству симметрии ( $S$-свойству):

$$
\begin{aligned}
& \frac{\partial}{\partial n_{+}} W^{\lambda}(z)=\frac{\partial}{\partial n_{-}} W^{\lambda}(z), \quad z \in \Delta, \\
& \frac{\partial}{\partial n_{+}} W^{\mu}(z)=\frac{\partial}{\partial n_{-}} W^{\mu}(z), \quad z \in \Gamma,
\end{aligned}
$$

где $\partial / \partial n_{+}$и $\partial / \partial n_{-}-$производные вдоль нормали справа и слева от кривых, а

$$
\begin{aligned}
& W^{\lambda}(z)=2 V^{\lambda}(z)-V^{\mu}(z)+\beta_{1} \operatorname{Re} z, \\
& W^{\mu}(z)=2 V^{\mu}(z)-V^{\lambda}(z)+\left(\beta_{2}-\beta_{1}\right) \operatorname{Re} z .
\end{aligned}
$$


§3. Доказательство теоремы.

Метод матричной задачи Римана-Гильберта

3.1. Функции второго рода и соотношения ортогональности. Введем функции второго рода

$$
r_{\vec{n}, j}(z)=\frac{n^{-\alpha}}{2 \pi i} \int_{0}^{\infty} \frac{l_{\vec{n}}(x) x^{\alpha} e^{-\beta_{j} x} d x}{x-z}, \quad j=1,2
$$

которые определены с точностью до нормировки $l_{\vec{n}}(x)$. Пусть $\gamma-$ произвольная кривая вокруг положительной полупрямой с горизонтальными асимптотами в бесконечности (см. рис. 7 ). В следующем предложении показано, что функция $r_{\vec{n}, 1}$ удовлетворяет соотношениям ортогональности на $\gamma$.

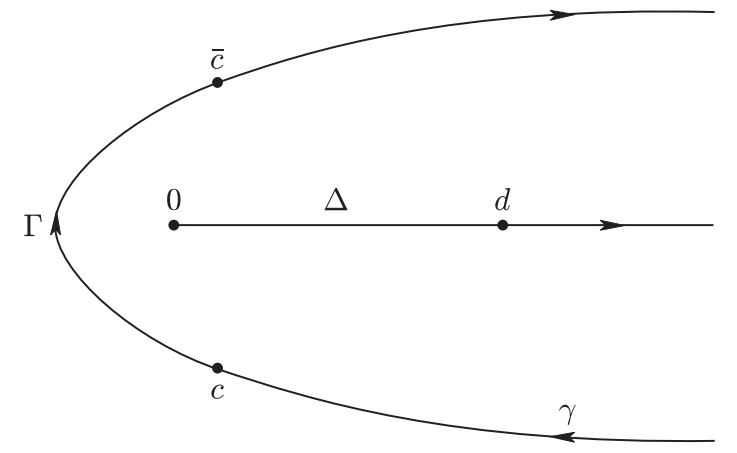

Рис. 7. Контур $\mathbb{R}_{+} \cup \gamma$

ПРЕДЛОЖЕНИЕ 3.1. Имеем

$$
\int_{\gamma} r_{\vec{n}, 1}(z) z^{k} e^{-\left(\beta_{2}-\beta_{1}\right) z} d z=n^{-\alpha} \int_{0}^{\infty} l_{\vec{n}}(x) x^{k+\alpha} e^{-\beta_{2} x} d x, \quad k \in \mathbb{Z}_{+}
$$

В частности,

$$
\int_{\gamma} r_{\vec{n}, 1}(z) z^{k} e^{-\left(\beta_{2}-\beta_{1}\right) z} d z=0, \quad k=0, \ldots, n_{2}-1 .
$$

ДоКАЗАТЕЛЬСтво. Подставляя выражение (3.1) для $r_{\vec{n}, 1}$ в интеграл в левой части (3.2) и используя теорему Фубини, получаем

$$
n^{-\alpha} \int_{0}^{\infty}\left(\frac{1}{2 i \pi} \int_{\gamma} \frac{z^{k} e^{-\left(\beta_{2}-\beta_{1}\right) z}}{x-z} d z\right) l_{\vec{n}}(x) x^{\alpha} e^{-\beta_{1} x} d x .
$$

По формуле Коши, примененной к функции $z^{k} e^{-\left(\beta_{2}-\beta_{1}\right) z}$ при $k \geqslant 0$, последнее выражение равно

$$
n^{-\alpha} \int_{0}^{\infty} l_{\vec{n}}(x) x^{k+\alpha} e^{-\beta_{2} x} d x .
$$


Это дает (3.2). Далее, (3.3) получаем из соотношений ортогональности (1.8) для полиномов Лагерра (при $r=2$ и $j=2$ ).

Рассмотрим также функцию, естественным образом связанную с системой Никишина (см. [8]):

$$
\widetilde{r}_{\vec{n}, 2}(z)=\frac{1}{2 \pi i} \int_{\gamma} \frac{r_{\vec{n}, 1}(x) e^{-\left(\beta_{2}-\beta_{1}\right) x} d x}{x-z} .
$$

Указанные функции второго рода удовлетворяют следующим интерполяционным условиям в бесконечности, эквивалентным соотношениям ортогональности (1.8):

$$
r_{\vec{n}, j}(z)=O\left(\frac{1}{z^{n_{j}+1}}\right), \quad j=1,2, \quad \widetilde{r}_{\vec{n}, 2}(z)=O\left(\frac{1}{z^{n_{2}+1}}\right)
$$

при $z \rightarrow \infty$.

3.2. Постановка задачи Римана-Гильберта. Рассмотрим следующую матричную задачу Римана-Гильберта:

1) $Y(z)-(3 \times 3)$ матричнозначная функция, аналитическая в $\mathbb{C} \backslash\left(\mathbb{R}_{+} \cup \gamma\right)$, имеюшая непрерьвные граничные значения слева и справа на ориентированных кривых $\mathbb{R}_{+}$и $\gamma$, связанные матрицей скачка (см. п. 2)); поведение $Y(z)$ в окрестности бесконечности и концевой точки контура (точки 0) задается в пाп. 3) и 4$)$;

2) $Y(z)$ имеет скачок на $\mathbb{R}_{+} \cup \gamma$ в виде $Y_{+}=Y_{+} J_{Y}$, где

$$
\begin{array}{ll}
J_{Y}(z)=\left(\begin{array}{ccc}
1 & z^{\alpha} e^{-\beta_{1} n z} & 0 \\
0 & 1 & 0 \\
0 & 0 & 1
\end{array}\right), & z \in \mathbb{R}_{+}, \\
J_{Y}(z)=\left(\begin{array}{ccc}
1 & 0 & 0 \\
0 & 1 & e^{-\left(\beta_{2}-\beta_{1}\right) n z} \\
0 & 0 & 1
\end{array}\right), & z \in \gamma ;
\end{array}
$$

3) $Y(z)$ имеет следующее поведение в окрестности бесконечности:

$$
Y(z)=\left(I+O\left(\frac{1}{z}\right)\right)\left(\begin{array}{ccc}
z^{2 n} & 0 & 0 \\
0 & z^{-n} & 0 \\
0 & 0 & z^{-n}
\end{array}\right), \quad z \rightarrow \infty, \quad z \in \mathbb{C}
$$

4) $Y(z)$ имеет следующее поведение в окрестности начала координат:

$$
Y(z)=O\left(\begin{array}{lll}
1 & h(z) & 1 \\
1 & h(z) & 1 \\
1 & h(z) & 1
\end{array}\right), \quad z \rightarrow 0, \quad h(z)= \begin{cases}|z|^{\alpha}, & \text { если }-1<\alpha<0 \\
\log |z|, & \text { если } \alpha=0 ; \\
1, & \text { если } 0<\alpha,\end{cases}
$$

где условие $O$ понимается поэлементно. 
ПРЕДЛОЖЕНИЕ 3.2. Решение этой задачи единственно и равно

$$
Y(z)=\left(\begin{array}{ccc}
L_{n}(z) & R_{n, 1}(z) & \widetilde{R}_{n, 2}(z) \\
l_{(n-1, n)}(n z) & r_{(n-1, n), 1}(n z) & \widetilde{r}_{(n-1, n), 2}(n z) \\
l_{(n, n-1)}(n z) & r_{(n, n-1), 1}(n z) & \widetilde{r}_{(n, n-1), 2}(n z)
\end{array}\right),
$$

где $L_{n}(z)$ есть полиортогональный полином Лагерра со стариим коэффициентом единича (1.10),

$$
R_{n, 1}(z)=r_{(n, n), 1}(n z), \quad \widetilde{R}_{n, 2}(z)=\widetilde{r}_{(n, n), 2}(n z),
$$

$r_{(n, n), 1} u \widetilde{r}_{(n, n), 2}$ заданы интегралами (3.1) и (3.4), соответствующими полиномам Лагерра $l_{(n, n)}$ со стариим коэффичиентом $n^{-2 n}$, а нормировка полиномов $l_{(n-1, n)}$ и $l_{(n, n-1)}$ выбрана таким образом, чтобь

$$
z^{n} r_{(n-1, n), 1}(n z) \rightarrow 1, \quad z^{n} \widetilde{r}_{(n, n-1), 2}(n z) \rightarrow 1
$$

при $z \rightarrow \infty$.

ДокАзАтЕльство. Проверим, что $Y(z)$, заданная (3.9), решает задачу Римана-Гильберта. Очевидно, $Y(z)$ аналитична вне $\mathbb{R}_{+} \cup \gamma$. Условия скачка на $\mathbb{R}_{+}$для первой и третьей строк $Y$ тривиальны, т.е. эти строки аналитичны на $\mathbb{R}_{+}$. Для элементов второй строки условия скачка записываются в виде

$$
\left(Y_{k, 2}\right)_{+}(z)=z^{\alpha} e^{-n \beta_{1} z}\left(Y_{k, 1}\right)_{-}(z)+\left(Y_{k, 2}\right)_{-}(z)
$$

которые вытекают из формулы Сохоцкого-Племеля. Аналогично проверяются условия скачка $Y(z)$ на $\gamma$. Поведение $Y(z)$ в бесконечности следует из условий нормальности индексов и условий интерполящии (3.5). Поведение $Y(z)$ в окрестности 0 легко проверяется (см. [9]).

Докажем теперь единственность решения задачи Римана-Гильберта. Пусть $Y(z)$ - какое-нибудь решение. Покажем, что оно равно (3.9). Первая строка $Y(z)$ не имеет скачка на $\mathbb{R}_{+} \cup \gamma$. Из поведения матрицы $Y(z)$ в 0 следует, что первая строка состоит из целых функций, а из поведения $Y(z)$ в бесконечности следует, что это полиномы степеней не больше $(2 n, 2 n-1,2 n-1)$. Далее, из формулы Сохоцкого-Племеля, условия скачка на контуре $\mathbb{R}_{+}$и поведения $Y(z)$ в нуле вытекает, что вторая строка есть преобразование Коши первой. Из тех же соображений, примененных к контуру $\gamma$, следует, что третья строка - преобразование Коши второй. Поведение второй и третьей строк в бесконечности дают интерполяционные условия (3.5), которые, как уже отмечалось, равносильны определению полиномов Лагерра (1.8). Таким образом, (3.9) - единственное решение задачи Римана-Гильберта.

Метод доказательства формул сильной асимптотики ортогональных полиномов, основанньй на рассмотрении матричной краевой задачи, был предложен П. Дейфтом и Х. Чжу в работе [6]. Следуя этому подходу, мы посредством цепочки обратимых преобразований $Y \rightarrow U \rightarrow T \rightarrow S$ придем к некоторой задаче Римана-Гильберта для матричной функции $S$. Матрица скачка этой функции $J_{S}$ будет иметь равномерную асимптотику на соответствуюшей системе контуров $\Sigma_{S}$ вида $J_{S}(z)=I+O(1 / n), z \in \Sigma_{S}$. Отсюда следует, что и сама матрица $S$ асимптотически равна $S(z)=I+O(1 / n)$ равномерно на компактах из $\overline{\mathbb{C}}$. Обращая цепочку преобразований, получаем асимптотику $Y$ и, в частности, асимптотику ортогональных полиномов Лагерра. 
3.3. Нормировка задачи Римана-Гильберта. Целью первого преобразования является нормировка матричной задачи Римана-Гильберта в бесконечности. Для этого от краевой задачи на $Y$ мы перейдем к задаче на $U$, решение которой будет вести себя как единичная матрица в окрестности $z=\infty$. Рассмотрим матричную функцию

$$
U(z)=C^{n} Y(z) \widetilde{\Phi}^{-n}(z)
$$

где

$$
\widetilde{\Phi}(z)=\operatorname{diag}\left(\Phi_{0}(z), \Phi_{1}(z) e^{-\beta_{1} z}, \Phi_{2}(z) e^{-\beta_{2} z}\right), \quad C=\operatorname{diag}\left(1, c_{1}, c_{2}\right) .
$$

Матричная функция $U(z)$ голоморфна в $\mathbb{C} \backslash\left(\mathbb{R}_{+} \cup \gamma\right)$, так как ветви $\Phi_{j}(z)$ голоморфны в $\Delta \cup \Gamma$ и не имеют нулей. Учитывая поведение $\Phi_{j}(z)$ в окрестности бесконечности, заключаем, что

$$
U(z)=I+O\left(\frac{1}{z}\right), \quad z \rightarrow \infty,
$$

т.е. $U(z)$ нормирована в бесконечности. Так как $\Phi_{j}(z)$ ограничены сверху и снизу в окрестности нуля, то $U(z)$ имеет то же поведение в нуле, что и $Y(z)$. Функции скачка имеют вид:

$$
\begin{aligned}
& J_{U}=\left(\begin{array}{ccc}
\left(\frac{\Phi_{0-}}{\Phi_{0+}}\right)^{n} & z^{\alpha} & 0 \\
0 & \left(\frac{\Phi_{1-}}{\Phi_{1+}}\right)^{n} & 0 \\
0 & 0 & 1
\end{array}\right), \quad z \in \Delta, \\
& J_{U}=\left(\begin{array}{ccc}
1 & z^{\alpha}\left(\frac{\Phi_{0}}{\Phi_{1}}\right)^{n} & 0 \\
0 & 1 & 0 \\
0 & 0 & 1
\end{array}\right), \quad z \in \mathbb{R}_{+} \backslash \Delta \\
& J_{U}=\left(\begin{array}{ccc}
1 & 0 & 0 \\
0 & \left(\frac{\Phi_{1-}}{\Phi_{1+}}\right)^{n} & 1 \\
0 & 0 & \left(\frac{\Phi_{2-}}{\Phi_{2+}}\right)^{n}
\end{array}\right), \quad z \in \Gamma, \\
& J_{U}=\left(\begin{array}{ccc}
1 & 0 & 0 \\
0 & 1 & \left(\frac{\Phi_{1}}{\Phi_{2}}\right)^{n} \\
0 & 0 & 1
\end{array}\right), \quad z \in \gamma \backslash \Gamma .
\end{aligned}
$$

Итак, функция $U$ является единственным решением следуюшей задачи РиманаГильберта:

1) $U(z)$ аналитична в $\mathbb{C} \backslash\left(\mathbb{R}_{+} \cup \gamma\right)$;

2) $U(z)$ имеет непрерывные граничные значения на $\mathbb{R}_{+} \cup \gamma$ такие, что $U_{+}=$ $U_{-} J_{U}$; 
3) $U(z)$ имеет следующее поведение в окрестности бесконечности:

$$
U(z)=I+O\left(\frac{1}{z}\right), \quad z \rightarrow \infty, \quad z \in \mathbb{C}
$$

4) $U(z)$ имеет то же поведение в нуле, что и $Y(z)$, см. (3.8).

Матрица скачка $J_{U}(z)$ с геометрической скоростью стремится к единичной при $n \rightarrow \infty$ для $z \in \mathbb{R}_{+} \backslash \Delta$ и $z \in \gamma \backslash \Gamma$. При $z \in \Delta$ и $z \in \Gamma$ матрица $J_{U}(z)$ имеет осциллирующие диагональные элементы, но допускает удачную факторизацию, которая лежит в основе следующего преобразования.

3.4. Факторизация матрицы скачка. Раскрытие линзы. Второе преобразование основано на следующей факторизации матрицы скачка $J_{U}(z)$ на отрезке $\Delta$ и аналогичной на кривой $\Gamma$ :

$$
\begin{aligned}
\left(\begin{array}{ccc}
\left(\frac{\Phi_{0-}}{\Phi_{0+}}\right)^{n} & z^{\alpha} & 0 \\
0 & \left(\frac{\Phi_{1-}}{\Phi_{1+}}\right)^{n} & 0 \\
0 & 0 & 1
\end{array}\right)= & \left(\begin{array}{ccc}
1 & 0 & 0 \\
z^{-\alpha}\left(\frac{\Phi_{1-}}{\Phi_{0-}}\right)^{n} & 1 & 0 \\
0 & 0 & 1
\end{array}\right)\left(\begin{array}{ccc}
0 & z^{\alpha} & 0 \\
-z^{-\alpha} & 0 & 0 \\
0 & 0 & 1
\end{array}\right) \\
& \times\left(\begin{array}{ccc}
1 & 0 & 0 \\
z^{-\alpha}\left(\frac{\Phi_{1+}}{\Phi_{0+}}\right)^{n} & 1 & 0 \\
0 & 0 & 1
\end{array}\right)
\end{aligned}
$$

Как видим, центральная матрица не зависит от $n$, а первая и последняя матрицы допускают аналитическое продолжение в области, расположенные соответственно справа и слева от отрезка $\Delta$. Введем дополнительные контуры. Пусть $\Delta_{+}$и $\Delta_{-}-$ дуги, соединяющие концы отрезка $\Delta$ и лежащие соответственно слева и справа от $\Delta$ в области, где $\left|\Phi_{0}\right|<\left|\Phi_{1}\right|$. Аналогично, $\Gamma_{+}$и $\Gamma_{-}-$дуги, соединяющие концы $\Gamma$ и лежашие соответственно слева и справа от $Г$ в области, где $\left|\Phi_{1}\right|<\left|\Phi_{2}\right|$ (см. рис. 8).

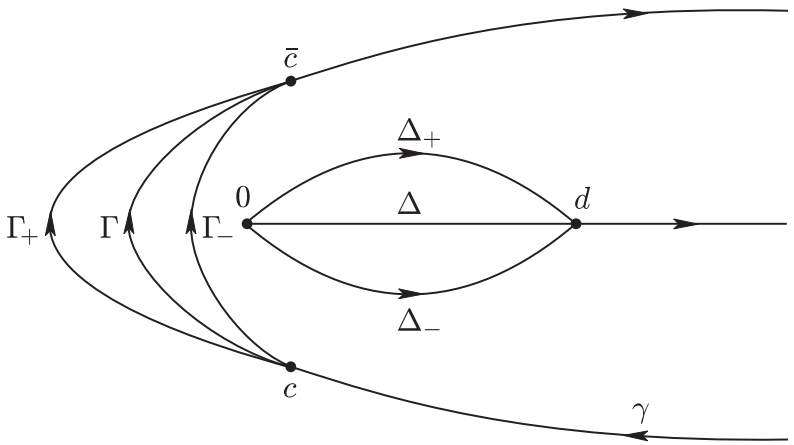

Рис. 8. Контур для $T$ 
Определим матрицу $T(z)$ следующим образом:

$$
\begin{aligned}
& T(z)=U(z)\left(\begin{array}{ccc}
1 & 0 & 0 \\
-z^{-\alpha}\left(\frac{\Phi_{1}}{\Phi_{0}}\right)^{n}(z) & 1 & 0 \\
0 & 0 & 1
\end{array}\right) \text { в области между } \Delta_{+} \text {и } \Delta \\
& T(z)=U(z)\left(\begin{array}{ccc}
1 & 0 & 0 \\
z^{-\alpha}\left(\frac{\Phi_{1}}{\Phi_{0}}\right)^{n} & 1 & 0 \\
0 & 0 & 1
\end{array}\right) \quad \text { в области между } \Delta_{-} \text {и } \Delta \\
& T(z)=U(z)\left(\begin{array}{ccc}
1 & 0 & 0 \\
0 & 1 & 0 \\
0 & -\left(\frac{\Phi_{2}}{\Phi_{1}}\right)^{n}(z) & 1
\end{array}\right) \quad \text { в области между } \Gamma_{+} \text {и } Г, \\
& T(z)=U(z)\left(\begin{array}{lrr}
1 & 0 & 0 \\
0 & 1 & 0 \\
0 & \left(\frac{\Phi_{2}}{\Phi_{1}}\right)^{n}(z) & 1
\end{array}\right) \quad \text { в области между } \Gamma_{-} \text {и } Г
\end{aligned}
$$

и $T(z)=U(z)$ в двух оставшихся областях.

Тогда легко проверить, что $T(z)$ является решением следуюшей задачи Римана-Гильберта:

1) $T$ аналитична в $\mathbb{C} \backslash\left(\mathbb{R}_{+} \cup \gamma \cup \Delta_{ \pm} \cup \Gamma_{ \pm}\right)$;

2) $T$ имеет следующие скачки на контурах:

$$
\begin{aligned}
& T_{+}(z)=T_{-}(z)\left(\begin{array}{ccc}
0 & z^{\alpha} & 0 \\
-z^{-\alpha} & 0 & 0 \\
0 & 0 & 1
\end{array}\right), \quad z \in \Delta \\
& T_{+}(z)=T_{-}(z)\left(\begin{array}{ccc}
1 & z^{\alpha}\left(\frac{\Phi_{1}}{\Phi_{0}}\right)^{-n} & 0 \\
0 & 1 & 0 \\
0 & 0 & 1
\end{array}\right), \quad z \in \mathbb{R}_{+} \backslash \Delta \text {, } \\
& T_{+}(z)=T_{-}(z)\left(\begin{array}{ccc}
1 & 0 & 0 \\
z^{-\alpha}\left(\frac{\Phi_{1}}{\Phi_{0}}\right)^{n} & 1 & 0 \\
0 & 0 & 1
\end{array}\right), \quad z \in \Delta_{ \pm}, \\
& T_{+}(z)=T_{-}(z)\left(\begin{array}{ccc}
1 & 0 & 0 \\
0 & 0 & 1 \\
0 & -1 & 0
\end{array}\right), \quad z \in \Gamma, \\
& T_{+}(z)=T_{-}(z)\left(\begin{array}{ccc}
1 & 0 & 0 \\
0 & 1 & \left(\frac{\Phi_{2}}{\Phi_{1}}\right)^{-n} \\
0 & 0 & 1
\end{array}\right), \quad z \in \gamma \backslash \Gamma,
\end{aligned}
$$




$$
T_{+}(z)=T_{-}(z)\left(\begin{array}{ccc}
1 & 0 & 0 \\
0 & 1 & 0 \\
0 & \left(\frac{\Phi_{2}}{\Phi_{1}}\right)^{n} & 1
\end{array}\right), \quad z \in \Gamma_{ \pm} ;
$$

3) $T(z)$ имеет следующее поведение в бесконечности:

$$
T(z)=I+O\left(\frac{1}{z}\right) ; \quad z \rightarrow \infty
$$

4) поведение $T(z)$ в окрестности начала координат зависит от $\alpha$ следующим образом:

$$
\begin{aligned}
& \text { при }-1<\alpha<0 \\
& T(z)=O\left(\begin{array}{lll}
1 & |z|^{\alpha} & 1 \\
1 & |z|^{\alpha} & 1 \\
1 & |z|^{\alpha} & 1
\end{array}\right), \quad z \rightarrow 0,
\end{aligned}
$$

при $\alpha=0$

$$
T(z)=O\left(\begin{array}{ccc}
\log |z| & \log |z| & 1 \\
\log |z| & \log |z| & 1 \\
\log |z| & \log |z| & 1
\end{array}\right), \quad z \rightarrow 0
$$

при $0<\alpha$

$$
T(z)=\left\{\begin{array}{cc}
O\left(\begin{array}{ccc}
1 & 1 & 1 \\
1 & 1 & 1 \\
1 & 1 & 1
\end{array}\right), & z \rightarrow 0 \text { вне линзы; } \\
O\left(\begin{array}{lll}
|z|^{-\alpha} & 1 & 1 \\
|z|^{-\alpha} & 1 & 1 \\
|z|^{-\alpha} & 1 & 1
\end{array}\right), \quad z \rightarrow 0 \text { внутри линзы. }
\end{array}\right.
$$

Матрицы скачка $T(z)$ не зависят от $n$ на контурах $\Delta$ и $Г$ и стремятся к единичным с геометрической скоростью при $n \rightarrow \infty$ во внутренних точках остальных контуров. Чтобы осушествить переход к краевой задаче относительно $S$ со скачками, равномерно стремящимися к единичным, нам понадобится решить ряд вспомогательных задач.

3.5. Проблема Римана-Гильберта с не зависящими от $n$ скачками. Рассмотрим краевую задачу, имеюшую те же матрицы скачков, что и $T$ на контурах $\Delta$ и $\Gamma$, и не имеюшую других скачков (можно ожидать, что решение этой задачи дает основной вклад в асимптотику $T$ вне точек $0, d, c, \bar{c})$ :

1) $N(z)$ аналитична $\mathbb{C} \backslash(\Delta \cup \Gamma)$; 
2) $N(z)$ имеет такие же скачки, как и $T(z)$, на $\Delta$ и $\Gamma$ :

$$
\begin{array}{ll}
N_{+}(z)=N_{-}(z)\left(\begin{array}{ccc}
0 & z^{\alpha} & 0 \\
-z^{-\alpha} & 0 & 0 \\
0 & 0 & 1
\end{array}\right), & z \in \Delta \\
N_{+}(z)=N_{-}(z)\left(\begin{array}{ccc}
1 & 0 & 0 \\
0 & 0 & 1 \\
0 & -1 & 0
\end{array}\right), & z \in \Gamma
\end{array}
$$

3) $N(z)$ ведет себя в бесконечности как единичная матрица

$$
N(z)=I+O\left(\frac{1}{z}\right), \quad z \rightarrow \infty
$$

Мы решаем эту проблему аналогично [10] (см. также [11]). Идея состоит в том, чтобы свести матричную краевую задачу к трем скалярным, используя биективное отображение римановой поверхности на сферу $\psi$. Будем искать решение в виде

$$
N(z)=\left(\begin{array}{lll}
N_{0}\left(\psi_{0}(z)\right) & N_{0}\left(\psi_{1}(z)\right) & N_{0}\left(\psi_{2}(z)\right) \\
N_{1}\left(\psi_{0}(z)\right) & N_{1}\left(\psi_{1}(z)\right) & N_{1}\left(\psi_{2}(z)\right) \\
N_{2}\left(\psi_{0}(z)\right) & N_{2}\left(\psi_{1}(z)\right) & N_{2}\left(\psi_{2}(z)\right)
\end{array}\right) .
$$

Введем ориентированные кривые на сфере (см. рис. 9)

$$
\begin{array}{lll}
\Sigma_{1}^{-}=\psi_{1}^{-}(\Delta), & \Sigma_{1}^{+}=\psi_{1}^{+}(\overleftarrow{\Delta}), & \Sigma_{1}=\Sigma_{1}^{-} \cup \Sigma_{1}^{+} \\
\Sigma_{2}^{-}=\psi_{2}^{-}(\Gamma), & \Sigma_{2}^{+}=\psi_{2}^{+}(\overleftarrow{\Gamma}), & \Sigma_{2}=\Sigma_{2}^{-} \cup \Sigma_{2}^{+}
\end{array}
$$

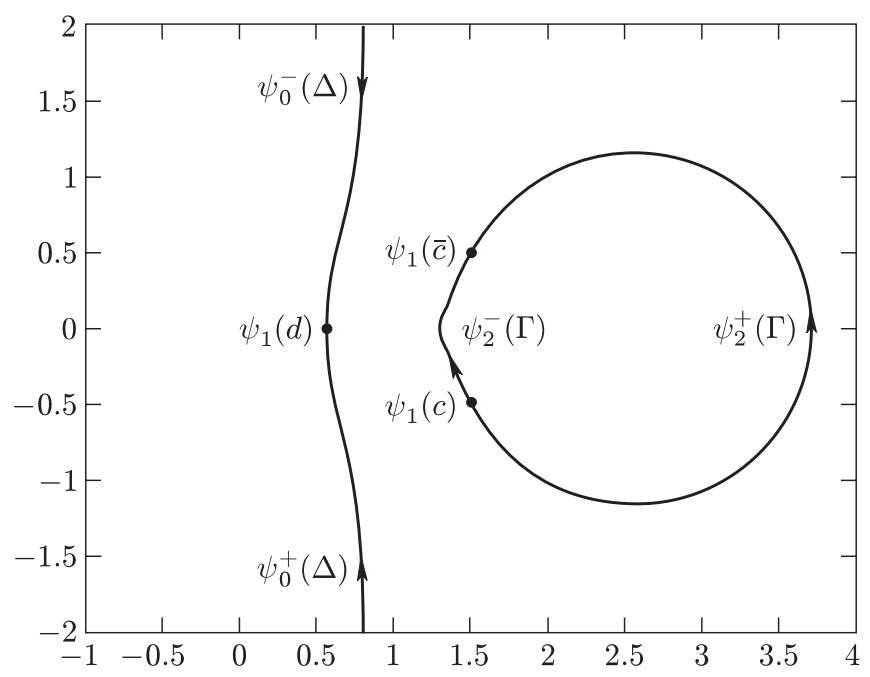

Рис. 9. Образ разрезов римановой поверхности при отображении $\psi$ 
Тогда матричная задача относительно $N$ перейдет в скалярные задачи для функций $N_{0}(\psi), N_{1}(\psi)$ и $N_{2}(\psi)$ :

1) $N_{j}$ аналитичны в $\mathbb{C} \backslash\left(\Sigma_{1} \cup \Sigma_{2}\right)$;

2) $N_{j}$ имеют скачки

$$
N_{j}^{+}(\psi)=N_{j}^{-}(\psi) \begin{cases}-z^{-\alpha}(\psi), & \psi \in \Sigma_{1}^{-} \\ z^{-\alpha}(\psi), & \psi \in \Sigma_{1}^{+} \\ -1, & \psi \in \Sigma_{2}^{-} \\ 1, & \psi \in \Sigma_{2}^{+}\end{cases}
$$

где $z(\psi)=2 / \psi-1 /\left(\psi-\beta_{1}\right)-1 /\left(\psi-\beta_{2}\right)$

3) $N_{j}$ имеют следующие нули и нормировку:

$$
\begin{array}{lll}
N_{0}(0)=1, & N_{0}\left(\beta_{1}\right)=0, & N_{0}\left(\beta_{2}\right)=0, \\
N_{1}(0)=0, & N_{1}\left(\beta_{1}\right)=1, & N_{1}\left(\beta_{2}\right)=0, \\
N_{2}(0)=0, & N_{2}\left(\beta_{1}\right)=0, & N_{2}\left(\beta_{2}\right)=1 .
\end{array}
$$

Решение этих задач легко находятся:

$$
\begin{aligned}
& N_{0}(\psi)=\frac{2^{\alpha-1 / 2} i\left(\psi-\beta_{1}\right)\left(\psi-\beta_{2}\right) g(\psi)}{\sqrt{D(\psi)}}, \\
& N_{1}(\psi)=\frac{\beta_{1}^{\alpha} \psi\left(\psi-\beta_{2}\right) g(\psi)}{\sqrt{D(\psi)}}, \\
& N_{2}(\psi)=\frac{\beta_{2}^{\alpha} \psi\left(\psi-\beta_{1}\right) g(\psi)}{\sqrt{D(\psi)}},
\end{aligned}
$$

где $D(\psi)=2\left(\beta_{1}+\beta_{2}\right) \psi^{3}-\left(\beta_{1}^{2}+8 \beta_{1} \beta_{2}+\beta_{2}^{2}\right) \psi^{2}+4\left(\beta_{1}+\beta_{2}\right) \beta_{1} \beta_{2} \psi-2 \beta_{1}^{2} \beta_{2}^{2}$ и

$$
g(\psi)= \begin{cases}\psi^{-\alpha} z^{-\alpha}, & z \text { слева от } \Sigma_{1} \\ \psi^{-\alpha}, & z \text { справа от } \Sigma_{1}\end{cases}
$$

3.6. Параметризация в окрестности концевых точек. Теперь матрицы скачков функции $T N^{-1}$ стремятся к единичным всюду кроме концевых точек $0, d$, $c, \bar{c}$. В этом пункте будут рассмотрены локальные краевые задачи в окрестностях этих точек. В окрестности точки 0 равновесная мера ведет себя как $1 / \sqrt{z}$. Параметризация в окрестности таких точек была построена в работе [9] с помощью функций Бесселя. В точке $d$, где равновесная мера ведет себя как $\sqrt{z-d}$, параметризация может быть построена аналогично работе [6] с помощью функций Эйри. Параметризация в окрестности точек $c$ и $\bar{c}$ проводится так же.

Пусть $U_{0}$ - достаточно маленькая окрестность нуля. Обозначим $\Delta_{0}=\Delta \cap U_{0}$, $\Delta_{0 \pm}=\Delta_{ \pm} \cap U_{0}$. Рассмотрим следуюшую краевую задачу в $U_{0}$ : 
1) $P_{0}$ аналитична в $U_{0} \backslash \Delta_{0} \cup \Delta_{0 \pm}$;

2) $P_{0}$ имеет такие же скачки на $\Delta_{0}$ и $\Delta_{0 \pm}$, как и $T$ :

$$
\begin{aligned}
& P_{0+}(z)=P_{0-}(z)\left(\begin{array}{ccc}
0 & z^{\alpha} & 0 \\
-z^{-\alpha} & 0 & 0 \\
0 & 0 & 1
\end{array}\right), \quad z \in \Delta_{0}, \\
& P_{0+}(z)=P_{0-}(z)\left(\begin{array}{ccc}
1 & 0 & 0 \\
z^{-\alpha}\left(\frac{\Phi_{1}}{\Phi_{0}}\right)^{n} & 1 & 0 \\
0 & 0 & 1
\end{array}\right), \quad z \in \Delta_{0+} \cup \Delta_{0-} ;
\end{aligned}
$$

3) $P_{0}$ имеет следующее поведение на границе $U_{0}$ :

$$
P_{0}(z)=N\left(I+O\left(\frac{1}{n}\right)\right), \quad n \rightarrow \infty \text { на } \partial U_{0}
$$

4) $P_{0}$ ведет себя в окрестности начала координат так же, как $T$ :

$$
\text { при } \alpha<0
$$

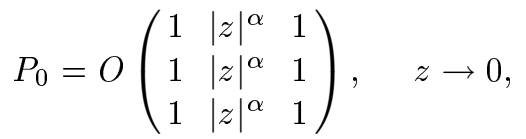

при $\alpha=0$

$$
P_{0}(z)=O\left(\begin{array}{lll}
\log |z| & \log |z| & 1 \\
\log |z| & \log |z| & 1 \\
\log |z| & \log |z| & 1
\end{array}\right), \quad z \rightarrow 0
$$

при $0<\alpha$

$$
P_{0}(z)=\left\{\begin{array}{l}
O\left(\begin{array}{ccc}
1 & 1 & 1 \\
1 & 1 & 1 \\
1 & 1 & 1
\end{array}\right), \quad z \rightarrow 0 \text { вне линзы; } \\
O\left(\begin{array}{lll}
|z|^{-\alpha} & 1 & 1 \\
|z|^{-\alpha} & 1 & 1 \\
|z|^{-\alpha} & 1 & 1
\end{array}\right), \quad z \rightarrow 0 \text { внутри линзы. }
\end{array}\right.
$$

Приведем решение этой задачи (доказательство аналогично [9]):

$$
P_{0}=E_{0} \widetilde{P}_{0} D_{0},
$$

где

$$
\begin{aligned}
E_{0}= & \frac{1}{2} N \operatorname{diag}\left((-z)^{\alpha / 2},(-z)^{-\alpha / 2}, 1\right)\left(\begin{array}{ccc}
1 & i & 0 \\
i & 1 & 0 \\
0 & 0 & 1
\end{array}\right) \\
& \times \operatorname{diag}\left(\sqrt{-\pi n \varphi}, \frac{1}{\sqrt{-\pi n \varphi}}, 1\right),
\end{aligned}
$$




$$
\begin{aligned}
& \widetilde{P}_{0}=\left\{\begin{array}{cc}
B\left(\begin{array}{ccc}
1 & 0 & 0 \\
-e^{-\alpha \pi i} & 1 & 0 \\
0 & 0 & 1
\end{array}\right) & \text { в области между } \Delta_{0} \text { и } \Delta_{0+} ; \\
B\left(\begin{array}{ccc}
1 & 0 & 0 \\
e^{\alpha \pi i} & 1 & 0 \\
0 & 0 & 1
\end{array}\right) & \text { в области между } \Delta_{0} \text { и } \Delta_{0-} ; \\
B & \text { в области между } \Delta_{0+} \text { и } \Delta_{0-},
\end{array}\right. \\
& B=\left(\begin{array}{ccc}
I_{\alpha}\left(-\frac{n \varphi}{2}\right) & -\frac{i}{\pi} K_{\alpha}\left(-\frac{n \varphi}{2}\right) & 0 \\
-n \pi i \varphi I_{\alpha}^{\prime}\left(-\frac{n \varphi}{2}\right) & -n \varphi K^{\prime}{ }_{\alpha}\left(-\frac{n \varphi}{2}\right) & 0 \\
0 & 0 & 1
\end{array}\right), \\
& D_{0}=\operatorname{diag}\left((-z)^{-\alpha / 2}\left(\frac{\Phi_{1}}{\Phi_{0}}\right)^{n / 2},(-z)^{\alpha / 2}\left(\frac{\Phi_{1}}{\Phi_{0}}\right)^{-n / 2}, 1\right) \text {, } \\
& \varphi=\ln \left(\frac{\Phi_{1}}{\Phi_{0}}\right) \text {. }
\end{aligned}
$$

Пусть теперь $U_{d}$ - достаточно малая окрестность точки $d$. Обозначим $\Delta_{d}=$ $\Delta \cap U_{d}, \Delta_{d \pm}=\Delta_{ \pm} \cap U_{d}, \widetilde{\Delta}_{d}=[d, \infty] \cap U_{d}$. Рассмотрим следуюшую краевую задачу в $U_{d}$ :

1) $P_{d}$ аналитична в $U_{d} \backslash \Delta_{d} \cup \Delta_{d \pm} \cup \widetilde{\Delta}_{d}$;

2) $P_{d}$ имеет такие же скачки на $\Delta_{d}, \Delta_{d \pm}$ и $\widetilde{\Delta}_{d}$, как и $T$ :

$$
\begin{aligned}
& P_{d+}(z)=P_{d-}(z)\left(\begin{array}{ccc}
0 & z^{\alpha} & 0 \\
-z^{-\alpha} & 0 & 0 \\
0 & 0 & 1
\end{array}\right), \quad z \in \Delta_{d} \\
& P_{d+}(z)=P_{d-}(z)\left(\begin{array}{ccc}
1 & 0 & 0 \\
z^{-\alpha}\left(\frac{\Phi_{1}}{\Phi_{0}}\right)^{n} & 1 & 0 \\
0 & 0 & 1
\end{array}\right), \quad z \in \Delta_{d \pm} \\
& P_{d+}(z)=P_{d-}(z)\left(\begin{array}{rrr}
1 & z^{\alpha}\left(\frac{\Phi_{1}}{\Phi_{0}}\right)^{-n} & 0 \\
0 & 1 & 0 \\
0 & 0 & 1
\end{array}\right), \quad z \in \widetilde{\Delta}_{d}
\end{aligned}
$$

3) $P_{d}$ имеет следующую асимптотику на границе $U_{d}$ :

$$
P_{d}(z)=N(z)\left(I+O\left(\frac{1}{n}\right)\right), \quad n \rightarrow \infty \text { на } \partial U_{d}
$$

Решение этой задачи может быть найдено по формуле (см. [6])

$$
P_{d}=E_{d} \widetilde{P}_{d} D_{d}
$$


где

$$
\begin{aligned}
& E_{d}=-\sqrt{\pi} e^{i \pi / 6} N \operatorname{diag}\left(z^{\alpha / 2}, z^{-\alpha / 2}, 1\right)\left(\begin{array}{ccc}
1 & 1 & 0 \\
i & -i & 0 \\
0 & 0 & 2 e^{-i \pi / 6}
\end{array}\right) \\
& \times \operatorname{diag}\left(\left(\frac{3}{2} n \varphi\right)^{1 / 6},\left(\frac{3}{2} n \varphi\right)^{-1 / 6}, 1\right),
\end{aligned}
$$

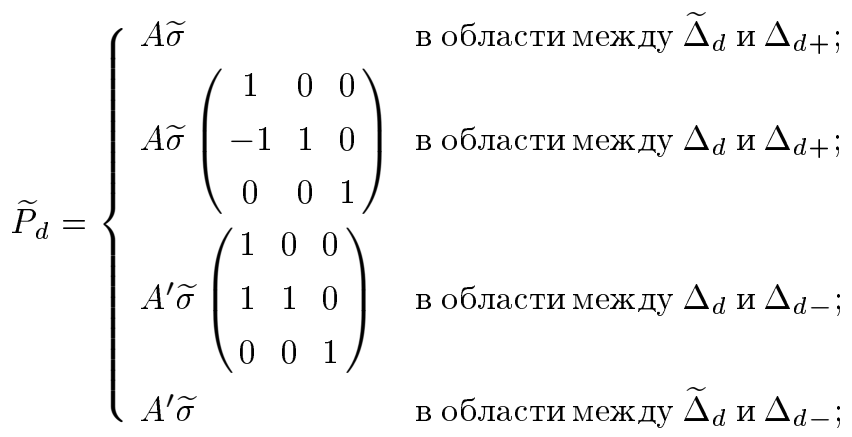

$$
\begin{aligned}
& A=\left(\begin{array}{ccc}
\operatorname{Ai}\left(\left(\frac{3}{2} n \varphi\right)^{2 / 3}\right) & \operatorname{Ai}\left(\varepsilon_{3}^{2}\left(\frac{3}{2} n \varphi\right)^{2 / 3}\right) & 0 \\
\operatorname{Ai}^{\prime}\left(\left(\frac{3}{2} n \varphi\right)^{2 / 3}\right) & \varepsilon_{3}^{2} \operatorname{Ai}^{\prime}\left(\varepsilon_{3}^{2}\left(\frac{3}{2} n \varphi\right)^{2 / 3}\right) & 0 \\
0 & 0 & 1
\end{array}\right), \\
& A^{\prime}=\left(\begin{array}{ccc}
\operatorname{Ai}\left(\left(\frac{3}{2} n \varphi\right)^{2 / 3}\right) & -\varepsilon_{3}^{2} \operatorname{Ai}\left(\varepsilon_{3}\left(\frac{3}{2} n \varphi\right)^{2 / 3}\right) & 0 \\
\operatorname{Ai}^{\prime}\left(\left(\frac{3}{2} n \varphi\right)^{2 / 3}\right) & -\mathrm{Ai}^{\prime}\left(\varepsilon_{3}\left(\frac{3}{2} n \varphi\right)^{2 / 3}\right) & 0 \\
0 & 0 & 1
\end{array}\right) \\
& \widetilde{\sigma}=\operatorname{diag}\left(e^{-i \pi / 6}, e^{i \pi / 6}, 1\right), \\
& D_{d}=\operatorname{diag}\left(z^{-\alpha / 2}\left(\frac{\Phi_{1}}{\Phi_{0}}\right)^{n / 2}, z^{\alpha / 2}\left(\frac{\Phi_{1}}{\Phi_{0}}\right)^{-n / 2}, 1\right) \\
& \varphi=\ln \left(\frac{\Phi_{1}}{\Phi_{0}}\right) \\
& \varepsilon_{3}=e^{2 \pi i / 3} \text {. }
\end{aligned}
$$

Краевые задачи для построения локальной параметризации в окрестностях точек $c$ и $\bar{c}$ формулируются и решаются так же, как в окрестности точки $d$.

3.7. Последнее преобразование. Окончание доказательства. Определим матричную функцию $S$ (см. рис. 10):

$$
S= \begin{cases}T N^{-1}, & z \notin \bigcup_{j \in\{0, d, c, \bar{c}\}} U_{j} ; \\ T P_{j}^{-1}, & z \in U_{j}, j \in\{0, d, c, \bar{c}\} .\end{cases}
$$




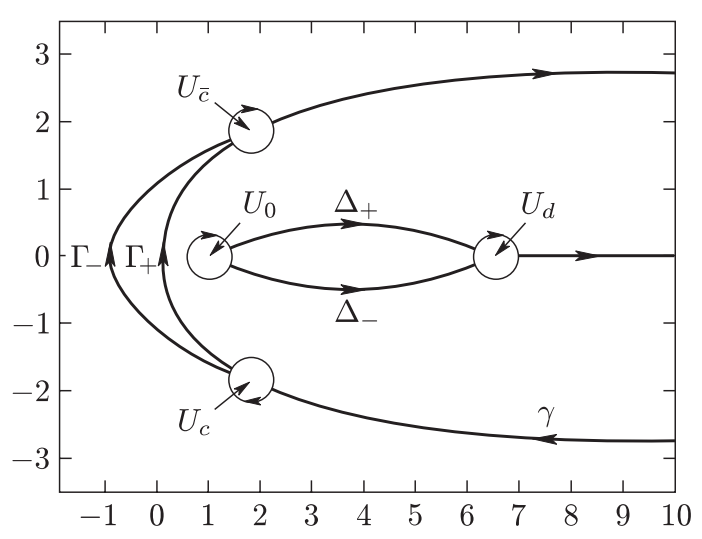

Рис. 10. Контур для $S$

Настояшая матрица имеет скачки, асимптотически равные единичным. Обрашая цепочку преобразований $Y \rightarrow U \rightarrow T \rightarrow S$, получаем утверждения основной теоремы.

\section{Список литературы}

1. Гончар A.A., Рахманов E. A. О сходимости совместных аппроксимаций Паде для системы функций марковского типа // Труды МИАН. 1981. Т. 157. С. 31-48.

2. Никишин E. M., Сорокин B. Н. Рациональные аппроксимации и ортогональность. М.: Наука, 1988.

3. Aptekarev A.I., Branquinho A., Van Assche W. Multiple orthogonal polynomials for classical weights // Trans. Amer. Math. Soc. 2003. V. 355. P. 3887-3914.

4. Сорокин В. Н. Сходимость совместных аппроксимаций Паде к функциям стилтьесовского типа // Изв. вузов. Сер. матем. 1987. № 7. С. 48-56.

5. Nuttall J. Asymptotics of diagonal Hermite-Padé polynomials // J. Approx. Theory. 1984. V. 42. № 4. P. 299-386.

6. Deift P., Zhou X. A steepest descent method for oscillatory Riemann-Hilbert problems. Asymptotics for MKdV equation // Ann. of Math. (2). 1993. V. 137. № 2. P. 295-368.

7. Van Assche W., Geronimo J.S., Kuijlaars A. B. J. Riemann-Hilbert problems for multiple orthogonal polynomials // Special functions 2000: Current perspective and future directions (Tempe, AZ) / ed. J. Bustoz et al. Dordrecht: Kluwer Acad. Publ., 2001. P. 23-59.

8. Гончар A.A., Рахманов E. А., Сорокин В.Н. Об аппроксимациях Эрмита-Паде для систем функций марковского типа // Матем. сб. 1997. Т. 188. № 5. С. 33-58.

9. Kuijlaars A.B.J., McLaughlin K.T.-R., Van Assche W., Vanlessen M. The Riemann-Hilbert approach to strong asymptotics for orthogonal polynomials on $[-1,1] / /$ Adv. Math. 2004. V. 188. P. 337-398.

10. Aptekarev A.I., Van Assche W. Scalar and matrix Riemann-Hilbert approach to the strong asymptotics of Padé approximants and complex orthogonal polynomials with varying weight // J. Approx. Theory. 2004. V. 129. P. 129-166.

11. Kuijlaars A.B.J., Van Assche W., Wielonsky F. Quadratic Hermite-Padé approximation to the exponential function: a Riemann-Hilbert approach // Constr. Approx. 2005. V. 21. № 3. P. 351-412.

Институт прикладной математики

имени М. В. Келдыша, г. Москва
Поступила в редакцию 28.03 .2005 и 14.10 .2005 